\title{
Principles of Formation of a Vehicle's Remote Diagnostics
}

\author{
Lukasz Muślewski ${ }^{1, *}$, Roman Zinko $^{2}$, Oleg Polishchuk ${ }^{3}$ \\ ${ }^{1}$ Faculty of Mechanical Engineering UTP in Bydgoszcz, Department of Vehicle Engineering, Al. \\ Prof. S.Kaliskiego 7, 85-796 Bydgoszcz, Poland \\ ${ }^{2}$ Lviv Polytechnik National University, St.Bandery Street, 12, Lviv, 79013, Ukraine \\ ${ }^{3}$ Khmelnitsky National University, Instytutska Street, 11, Khmelnytskyi, 29000, Ukraine
}

\begin{abstract}
Complex of remote diagnostics and control of an automobile's nodes enables to remotely control the state of vehicle's nodes and diagnose malfunctions; control ecological norms and compliance with traffic regulations. Its implementation will allow diagnostics of intermittent malfunctions due to application of the proposed methods of remote diagnostics of an automobile's nodes as well as to reduce terms of diagnostics significantly due to application of intellectual system of malfunctions detection and due to possibility to control parameters in real time mode. Implementation of the complex will cut unforeseen idle time of vehicles due to preventive control of the tendencies in nodes parameters changes, and due to executing modern repair or replacement of nodes and units. The complex grants safe local and remote access to data and reports by using WebPortal and VPN technologies with possibility to appoint personal right to every user. Due to application of the elaborated methods and the complex that implements it, one can achieve high rate of economic efficiency due to collection of statistic information under conditions of actual exploitation, diagnostics of emerging malfunctions of various difficulty degrees, and making necessary corrections in the structure of an automobile's nodes prior to serial production launch.
\end{abstract}

\section{Introduction}

Recently, the performance of vehicles, its power, economic, environmental and reliability indicators have increased significantly. The accelerated growth in the use of motor vehicles causes a further increase in the costs of their maintenance and repair. In this regard, the role of operational and accurate diagnostics in reducing operating costs will only increase.

The development of modern systems of several key automobile's nodes increases road safety, technical specifications and environmental safety standards. However, when complex modern car systems work together, a malfunction in one of the nodes can cause a whole complex of incorrect readings of conjugated electronic systems. Many malfunctions can manifest themselves in a time lag (the so-called alternative malfunctions). Effective diagnostics of such malfunctions is possible only in the active phase of malfunction manifestation, and is not always possible to be achieved by force. This greatly complicates the process of troubleshooting and calls for development and application of specialized methods and diagnostic tools.

Currently, most vehicle breakdowns occur on highways or city streets far from any service station. For the prevention of breakdowns, it is advisable to use remote diagnostic systems. Such systems make it possible to know information about the state of the functional nodes of a car in real time, thereby assessing its technical state.

\footnotetext{
* Corresponding author: Lukasz.Muslewski@utp.edu.pl
} 


\section{Problem statement}

Nowadays, two main trends in the development of automobile diagnostics are distinguished. The first trend is the location of technical diagnostic tools on the automobile itself. Means of technical diagnostics can be divided into three types according to their interaction with the object of diagnosis: built-in (included in the structure), external (not included in the structure), and installed on the automobile [1].

Diagnosis at service stations does not solve the problem of restoring the vehicle's serviceability, since its main task (to quickly (if necessary) service the car) is rather complex in this case.

In addition, the use of stationary and portable diagnostics tools is usually associated with the operations of connecting, adjusting and removing sensors and connected accessories, which leads to large labor costs for supplementary work. On average, they make up from 80 to $85 \%$ of the time of the total amount of work on the diagnostics.

These drawbacks are largely eliminated by the introduction of an integrated diagnostic system. Built-in diagnostics can be implemented in two ways: by installing special control sensors built into various vehicle systems with signals output to control points; by equipping a car with sensors and additional measuring instruments that provide continuous information on the technical state of the systems, components and units of the car.

The second trend is remote diagnostics. Car manufacturing companies started to equip their cars with Internet access points, which, along with navigation, allowed to enter in the database addresses of specific dealerships where this car can be serviced. Clients have a possibility of prompting for an emergency notification, when it is time for the next scheduled maintenance; they do not need to look for the dealer's coordinates on the network, they just start navigation that shows the location of the nearest authorized center.

After the client's call, the diagnostician can immediately make a request from his workplace to the car and get information about error codes, view parameters and even start activation in order to prepare for submitting the car to a car service.

\section{Analysis of recent research and publications}

Chevrolet has launched a system called "Proactive Alerts" on some of its vehicles [2]. This system scans all nodes of the car at startup and, when errors are detected, shows notifications about them on the driver's multimedia screen. However, the main difference between the system and Chevrolet is that, theoretically, with certain special settings, it can quickly send an e-mail message about possible errors, which is already enough for technical services to monitor the state of the car in real-time. So far, it can only be installed on the Silverado, Tahoe, Suburban and Corvette models of the last year range. The company does not deny further development of this system, its distribution and implementation in a wider range of models.

Scania experimentally installs the "Scania Remote Diagnostics" system [3] on tractors. Also, "Scania Communicator", official licensed services, allow specialists to examine all the inside components of the vehivle and pre-order the required spare parts, which will significantly reduce maintenance time, and, respectively, the time saved can be used more efficiently. The related ScaniaAssistance service allows you to track and promptly call qualified specialists in case of such breakdowns when the car cannot move independently.

The main idea of remote diagnostics is to integrate telematic systems into existing information infrastructures (cellular or radio) [4]. Currently, there are a number of systems for remote monitoring of hardware using telematics equipment. These systems are used to control vehicles in various fleets, as well as for single vehicles that meet the stated requirements. Currently, the following systems operate successfully: JDLink system, USA, DEERE \& COMPANY - "Remote optimization of vehicles work and logistics" and SCANIA FLEX system, Sweden, Scania AB - "Personal flexible maintenance plan" [5-8]. 
These systems have a wide range of capabilities that allow remote diagnostics of vehicles, but these systems are only compatible with vehicles produced by these concerns.

As a remote diagnostics, the Onstar service functionality from GeneralMotors is used, which are independent means of diagnostics, but are still used as supplementary equipment along with tools from the manufacturer.

Daimler (dealer auto scanner) offers the "Virtual Assistant" service, which is a system for transmitting data about the state of the car and remote diagnostics in real time.

\section{Presenting mainmaterial}

The diagnostics complex (Fig. 1) receives signals from the vehicle that display its technical state, namely, operating characteristics, registration data, and its functional nodes. The complex recognizes the incoming data, monitors the level of performance degradation, detects probable malfunctions, and sends a signal to the vehicle with an assessment of the technical state of the vehicle and its functional nodes, as well as recommendations to adjust the characteristics to optimal ones. Information is transmitted between the vehicle and the complex by means of telecommunication. The signals transmitting operational characteristics of the car and its functional nodes are received from the controllers through the test connector of the car.

Companies that are interested in the development of car maintenance create a technical support service to work with clients via the Internet. They create a websites containing software libraries and databases on technical specifications, troubleshooting, availability of spare parts and addresses of repair enterprises, etc.

Car owners purchase an adapter that connects the test connector to the modem, a cell phone (smartphone) and / or additionally connect the adapter to the laptop. Car owners are registered in an automated diagnostic system. They receive the necessary software for their car from the technical support service.

Car owners perform diagnostics using a local system in the car; if necessary, go online and select the type of service.

With the help of the software using the database available on the server, car diagnostics is performed and appropriate instructions are given to the car owner.

For the lower level of this system (a local automatic vehicle malfunction diagnostics system), a design is being developed in the form of an interface unit connected to a computer. Programs for the microcontroller of the interface unit and computer have been developed.

In most modern cars, there are multiplex data buses, through which data is exchanged with various car self-diagnostics systems. There may be several of them, different in speed, protocol and set of transmitted data. The configuration and tire protocols depend on the level of development of the car's self-diagnostics systems.

Most communication protocols (CAN, Lin, DeviceNet, CANopen, SAE J1939) [9-14] and adapters are supported by the WarwickControlTechnologies X-Analyser software. When using free version one can see information transmitted via the bus on the display in numerical form.

An example of a multiplex data bus is the CAN bus. CAN communication protocol is standardized according to ISO 11898-1 (2003). This standard mainly describes a data exchange layer consisting of a logical control (LLC) subsection and an access control (MAC) subsection, and some aspects of the physical layer of the ISO / OSI model. The remaining layers of the protocol are left to the discretion of the network developer [15]. CAN benefits:

- affordability;

- protocol realization on hardware level;

- primitive transmission line; 
- superb ability to detect errors and malfunctions as well as their localization;

- malfunctions detection and check system.

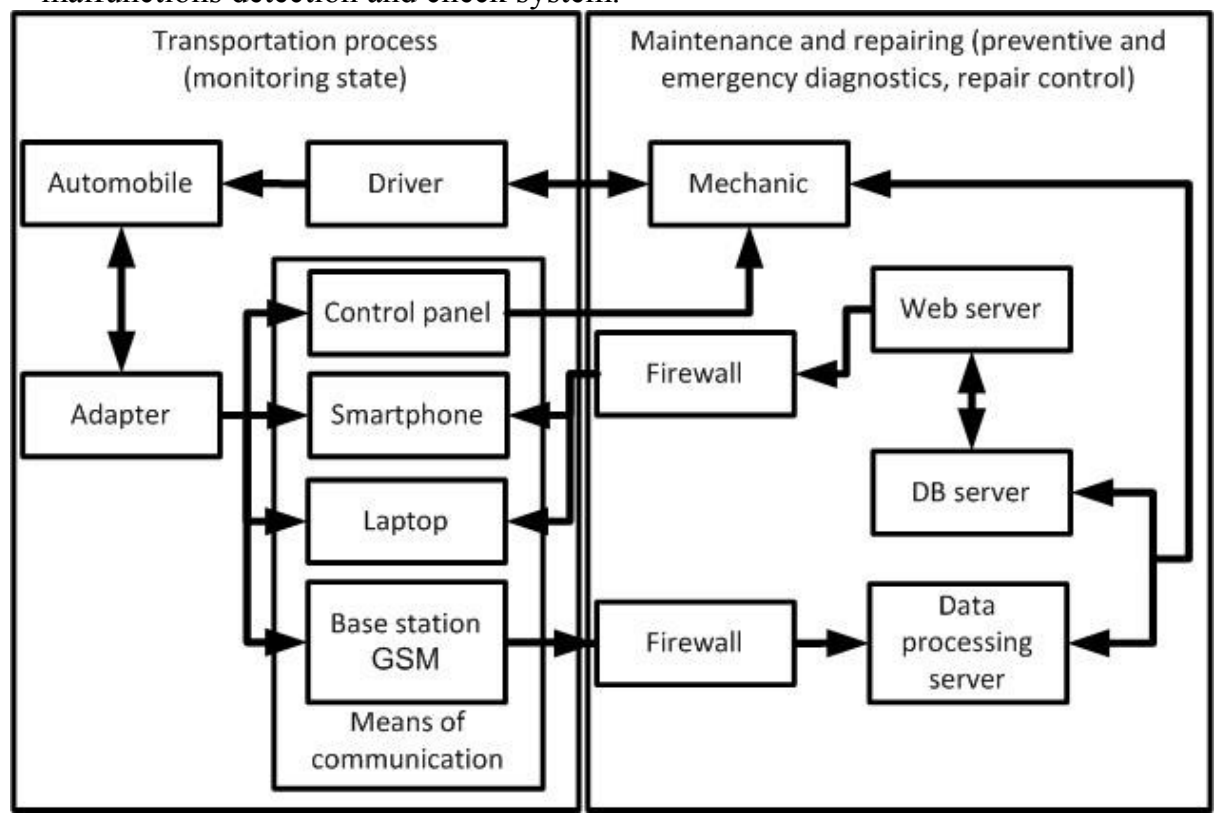

Fig. 1. Complex of remote diagnostics and control of the state of an automobile's nodes.

CAN is a system based on a serial bus with multifunctional capabilities; all CAN nodes are capable of transmitting data, and some CAN nodes can request a bus at the same time. The transmitter transmits a message to all CAN nodes. Each node, based on the identifier obtained, determines whether it should process the message or not. The identifier also determines the priority that the message has when accessing the bus [9].

Modern cars have electronic systems, operation of which is based on the parameters of the car controlled by a set of sensors on various nodes that can signal an error in their work with a specific diagnostic code of the self-diagnostics system. When several such systems interact, a malfunction of one of the parameters can cause incorrect operation of several systems at the same time. At the moment of error, each of the systems signals the code of the alleged cause. Error diagnostics can be greatly reduced by obtaining important information from the relevant codes. Diagnostic standards have been developed to unify the interaction protocols and messages format of these systems.

On-board navigation equipment (Fig. 2) interacts with the main technological components of the technical state monitoring system of the vehicle within the framework of the ideology of a mobile information-diagnostics system. The signals from sensors and vehicle systems are processed, and then an array of messages is generated. To obtain information from the diagnostics control systems that are part of the nodes and units of the vehicle, the information-diagnostic system generates a series of necessary commands. Having received encoded information from the standard built-in control systems, the information-diagnostic system, using its own software, determines the codes and creates an array of diagnostic messages. The purpose of identifying codes is to recognize vehicle states that are dangerous for continuing further work, first of all, creating a risk of expensive repairs, accident, loss of a dealership guarantee and other unfortunate consequences.

When a malfunction occurs during movement, it calls for recognition of the signs of malfunctions and then makes various decisions about the possibility of continuing the 
movement. Diagnostics information received from the diagnostics port or from the sensors of the standard on-board diagnostics system is processed on board in an automated mode [16].

Diagnostics system with data processing software is the most flexible. It allows you to read OBD-II codes and data streams in real time and present them in an intuitively clear format, in numerical form, in a form of description of possible malfunctions, in a form of tables, as well as in graphical form, including a form of multiparameter graphs. Using such system, you can conduct virtual tests: manually change one of the parameters and see what happens to the rest. At the same time, the protocol necessary for a detailed analysis of transients is done in real time. Such protocols can be useful for routine diagnostics: you can gradually accumulate an "engine history" and identify probable problems in a timely manner. Such diagnostics system can be designed on the basis of wireless technologies for data transmission and remote analysis in a data center.

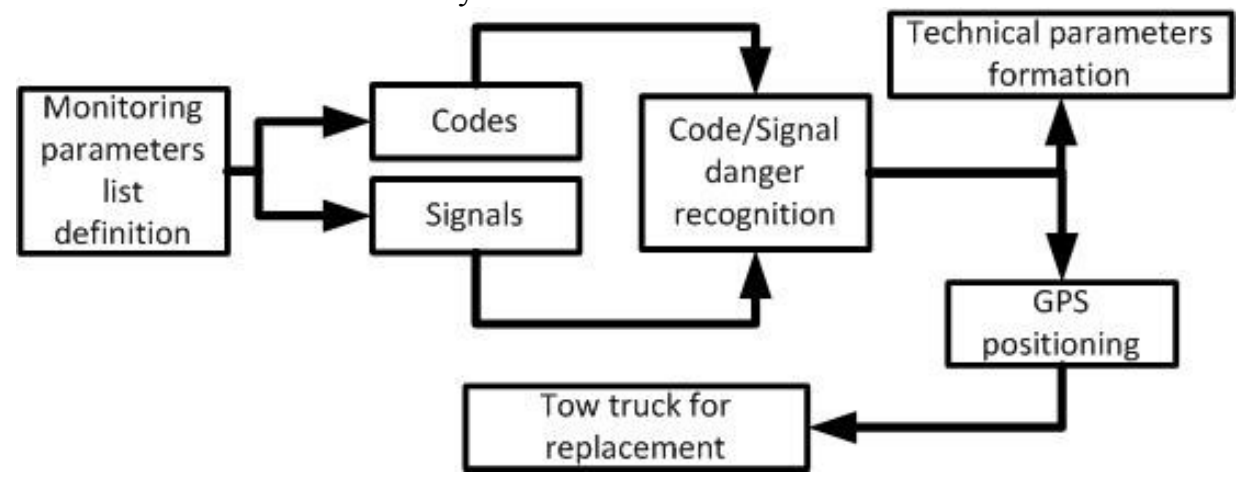

Fig. 2. Algorithm of vehicle's technical state monitoring system.

Currently, the most common wireless data transfer technologies are as follows:

- GPRS, EDGE (GSM standard);

- CDMA;

- WiFi.

More modern HSDPA technologies, WiMAX which are 3.5G-4G generation standards are in test operation. When comparing various wireless access technologies, the following points should be taken into account. First of all, the higher the frequency range, the smaller the communication range (cell radius) is, but the higher the data rate is. In this regard, for example, from the point of view of lowering capital expenditures in areas with low population density, it is preferable to build CDMA2000 networks in the $450 \mathrm{MHz}$ range, and to compare broadband wireless access technologies of large cities, CDMA 1x EV-DO networks in more high-frequency ranges (1.9 or $2.1 \mathrm{GHz})$ [17].

Existing diagnostics algorithms have an insufficient diagnostics level when analyzing the causes of complex and "intermittent" malfunctions, due to the lack of the possibility of applying real-time diagnostic techniques and the possibility of executing diagnostic actions at the main nodes, instantly receiving a system response to this effect exactly when the error is in the active phase.

To eliminate these shortcomings, we propose a method for remote diagnostics and monitoring of the state of an automobile's node (Fig. 3). A method for remote diagnostics consists in collecting diagnostic parameters at the monitoring object, initial processing and buffering, transfer of data using modern wireless technologies to a remote data processing server, and further analyzing and predicting technical state of the vehicle.

The method of remote diagnostics is described by remote diagnostics algorithms. Due to technical complexity of the systems of modern vehicles and the interpenetrating interaction of its nodes, there are malfunctions, the active phase of which is manifested by chance. 
Therefore, such intermittent malfunctions may not occur during local diagnostics directly at the service center. Diagnostics and determination of the cause of these malfunctions becomes impossible in the passive phase of the malfunction. Very often, all nodes of the vehicle work exclusively in normal modes and diagnostics by any method or equipment will not show any deviations. However, the malfunction will stay and may reappear when a combination of various external and internal factors cause it. The method of remote diagnostics allows remote monitoring of the safety of vehicles, as well as to fix the manifestation of the active phase of the malfunction and obtain important information during various diagnostics actions, and determine the true source of the malfunction.

One of the cyclic processes is the collection of sensor readings, primary diagnostics, formation of a data packet to transmit and forward information. This process can be configured based on the set priorities of the parameters of the collected information. This is necessary for the packet for transmission to be optimal, carry all the basic information, and do not contain redundant information. Based on the specifics of the application, a personal priority can be assigned to each of the transmitted parameters, which will affect the transmission of the parameter by means of wireless connection. On the part of the data center, the process of receiving, storing and analyzing information from diagnostic objects is cyclically active. The database is being constantly updated with the transmitted parameters, on the basis of which it is possible to obtain interconnected diagrams and trends in the vehicle parameters. Upon occurrence of an error, all basic information, if necessary, will be provided to the operator for further processing [6]. Having remote access to the information provided by the data processing server, a diagnostic engineer can:

- track the readings of car sensors in real time (on-line);

- receive current or previously recorded error codes from the self-diagnostics systems;

- conduct remote diagnostics based on the OBD-II standard (if this is supported by the vehicle's systems);

- receive data from sensors in diagnostics mode, for the analysis of transient processes with a frequency of $50-500 \mathrm{~Hz}$;

- get information on typical situations similar to the situations already recorded in the intelligent system to detect malfunctions;

- get information about the dynamics of changes in the parameters of the nodes and get the response of the system nodes when executing various diagnostics actions, immediately in the active phase of the manifestation of an intermittent malfuntion.

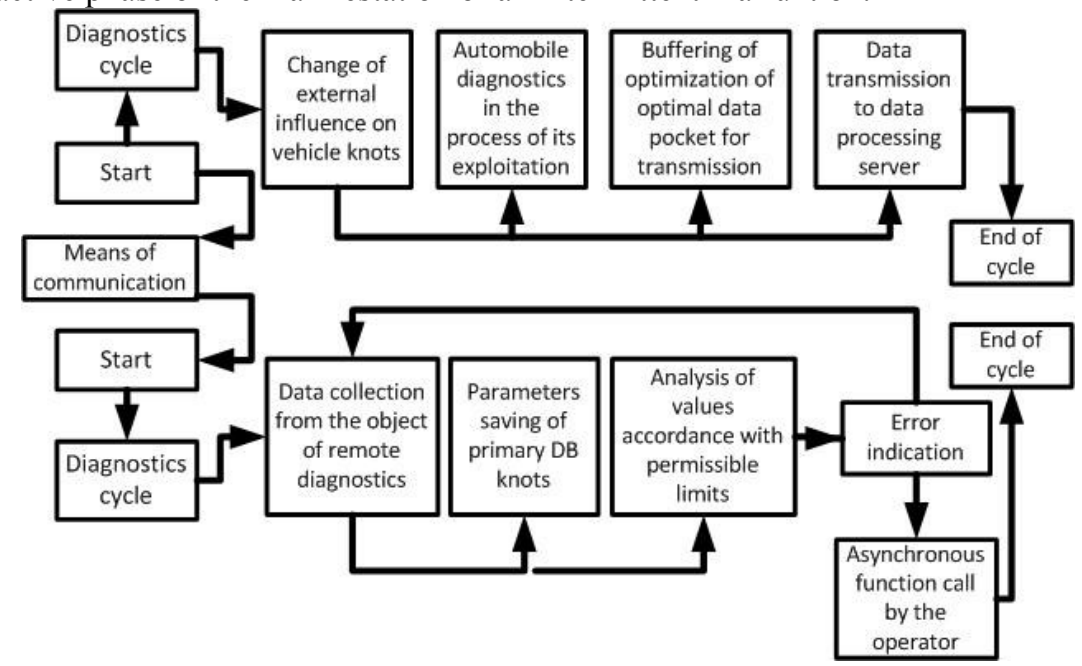

Fig. 3. Functional scheme of operation of assembly modules, receiving and transmission, data processing. 
The complex of remote diagnostics and control of the state of vehicle nodes implements the remote diagnostics technique, which enlists collection of diagnostics parameters at the object of control, initial processing and buffering, transfer of data via modern wireless technologies to a remote data processing server and further analysis by means of an intelligent error detection system, graphical analysis by various interpolation algorithms.

The hardware and software complex includes:

- the hardware diagnostics module, which is a device installed on the car and switched to the multiplex data bus through a test connector;

- data processing server, which is a service center that receives, stores and processes data from objects of control;

- software for processing data from objects of control, their analysis and assessment of the safe operation of vehicles on the basis of an intelligent system of malfunctions detection;

- prediction of vehicle's state, which defines the terms of malfunctions [18].

The complex has a modular structure that allows you to connect high-speed modules for wireless data transmission, based on modern EDGE, CDMA technologies etc., allowing you to implement a completely remote process for diagnostics of the state of the automobile's nodes. At the object of control, the operation modes of the complex can be observed on LEDs that reflect the state of the system (monitoring, diagnostics, malfunction). In the complex, control of the state of the automobile's nodes is carried out by control of the basic parameters of the node and analysis of diagnostic codes received from self-diagnostics systems. To solve the problems of managing the complex and providing a convenient interface for working with the received data, ESI tronic software can be used.

The software of the remote diagnostics complex can function under the control of Windows, Linux operating systems etc. The requirements for the hardware configuration of a computer are determined by the requirements of the operating system.

Prediction of the state of the vehicle is based on four types of initial information: statistics of repairs, expert assessment of the resource, statistics of diagnostics, data on technological loads. In this case, a diagnostics model is used.

Diagnostics parameter-forming model $[19,20]$ is a mathematical apparatus that uses data on the timing and results of the diagnostics performed in the process of its functioning. The peculiarity of this model is obtaining of the value of the residual resource of the unit based on data on the current technical state. Totality of output data for this model is presented as "Diagnostics statistics". This model can be represented by the formula (1):

$$
[Z, M, v]=f\left[Y\left(t_{i}\right) S\left(Y, t_{i}\right)\right]
$$

in which $Y\left(t_{i}\right)$ - value of the diagnosed parameter $Y_{i}$ for the exploited unit (current implementation) at the moment $t_{i}$;

$S\left(Y, t_{i}\right)$ - diagnostics data statistics on previous implementations.

In this model, only $t_{i}$ value is determined; it is described by the distribution law of Z, M and $\mathrm{v}$. The starting point of $t_{y}$ forecast forestalling is the time of the last diagnostics.

\section{Conclusions}

The complex of remote diagnostics and control of the state of an automobile's nodes will enable remote control of the state of vehicle node and troubleshooting, control compliance with environmental standards and traffic regulations. Its implementation will enable diagnostics of intermittent malfunctions due to application of the proposed method of remote diagnostics of an automobile's nodes, as well as significantly reduce time for 
diagnostics due to use of an intelligent system for detection of errors and the ability to control parameters in real time. Implementation of the complex will reduce unforeseen idle of vehicles due to preventive control of trends in the nodes parameters, and timely repair or replacement of nodes and units. The complex implements secure local and remote access to data and reports using WebPortal and VPN technologies, with the possibility to assign personal rights to each user.

Due to application of the elaborated methods and the complex that implements it, one can achieve high rate of economic efficiency due to collection of statistic information under conditions of actual exploitation, diagnostics of emerging malfunctions of various difficulty degrees, and making necessary corrections in the structure of an automobile's nodes prior to serial production launch.

\section{References}

1. The basic principles of diagnosing MSHT using modern diagnostic equipment / P.S. Sinitsin, H.D. Kokoriev, I.A. Uspenskyi // Collection of scientific works of students of the Ryazan State Agrotechnological University named after P.A. Kostychova. - Ryazan, - P. 263-269, (2011)

2. Proactive Alerts analyzes the condition of the car: https://petrenco.com/avto.php?txt=605/ (date of the application: 03.11.2019)

3. Extention useful life - with technology Scania Remote Diagnostics http://scanauto.ru/media-center-scania/news-scania/uvelichenie-vremeni-poleznogoispolzovaniyaavtomobilya-pri-pomowi-sistemy-udalennoj-diagnostiki-scania-remotediagnostics. (date of the application: 03.11.2019)

4. Fuks V.A. Universal system for remote vehicle diagnostics // Young scientist. - 2019. №12. - P. 40-44. - URL https://moluch.ru/archive/250/57481/ (date of the application: 03.11.2019)

5. Denton, T. (2012). Automobile electrical and electronic systems: Automotive technology: Vehicle maintenance and repair. NewYork: Routledge, (2012)

6. Pupkov K.A., Konkov V.G. Intelligent systems: research and creation. - M.: Publishing House of MSTU named after N.E. Bauman, 2003. - 345 p., (2003)

7. Website www.deere.com - company official website John Deere. (date of the application: 03.11.2019)

8. Website www.scania.com LLC «Scania Service " - The official dealer of the Swedish automotive concern Scania CV AB in Russia. (date of the application: 03.11.2019)

9. http://www.can-cia.org/ - Official website of an international organization CAN In Automation. (date of the application: 03.11.2019)

10. http://www.obdii.com/ - Official website of the international standardization organization OBD II. (date of the application: 03.11.2019)

11. Dzhelekarski P., Alexiev D. Initializing Communication to Vehicle OBDII System. Proc. Intern. Conf. Electronics, 2005, vol. 3, pp. 46-52., (2005)

12. Malekian R., Moloisane N.R., Nair L., Maharaj B.T., ChudeOkonkwo U.A.K. Design and Implementation of a Wireless OBD II Fleet Management System. IEEE Sensors J., 2017, vol. 17, no. 4, pp. 1154-1164, (2017)

13. Zhang H., Kang W. Design of the Data Acquisition System Based on STM32. Procedia Comp. Sc., 2013, vol. 17, pp. 222-228, (2013)

14. Si H., Aung Z.M. Position Data Acquisition from NMEA Protocol of Global Positioning System. IJCEE, 2011, vol. 3, no. 3, pp. 353-357, (2013)

15. Garanin M.V. Systems and networks of information transfer. - M.: Radio and communication, 2001-336 p., (2001) 
16. Principles of developing a telematic system for monitoring the technical condition of cars / S. V. Zhankaziev, S. P. Ignatiev // Bulletin of the Moscow Automobile and Road State Technical University (MADI). - 2011. - № 3. - P. 22-28, (2011)

17. Pljetnjev S.V. Engine of truck diagnostic on the basis of technology ODX / S.V. Pljetnjev, J.V. Krjukov, A.V. Ferenc // Announcer of the Kazan state technical university the name of A.N. Tupoljev. - 2014. - № 2, - P. 58-61.

18. Shakhnovich I.V. Modern wireless technology. - M.: Technosphere, 2006. - 288 p., (2014)

19. Aubekerov N.A. Individual assessment of operational damage to vehicle parts / N.A. Aubekerov, J.N. Aubekerova, A.N. Abatova // Automotive industry. - 2014. - № 11. P. 18-20., (2014)

20. M. Łukasiewicz, T. Kałaczyński, J. Musiał, J. Shalapko. Diagnostics of buggy vehicle transmission gearbox technical state based on modal vibrations, Journal of Vibroengineering, 16, 6, (2014) 